\title{
Classification of quantum groups and Belavin-Drinfeld cohomologies for orthogonal and symplectic Lie algebras
}

\author{
Boris Kadets, ${ }^{1}$ Eugene Karolinsky, ${ }^{1}$ lulia Pop, ${ }^{2}$ and Alexander Stolin ${ }^{3}$ \\ ${ }^{1}$ Department of Pure Mathematics, Kharkiv National University, Kharkiv, Ukraine \\ ${ }^{2}$ Department of Mathematical Sciences, Chalmers University of Technology, Gothenburg, \\ Sweden \\ ${ }^{3}$ Department of Mathematics, Gothenburg University, Gothenburg, Sweden
}

(Received 24 July 2015; accepted 8 May 2016; published online 25 May 2016)

\begin{abstract}
In this paper we continue to study Belavin-Drinfeld cohomology introduced in Kadets et al., Commun. Math. Phys. 344(1), 1-24 (2016) and related to the classification of quantum groups whose quasi-classical limit is a given simple complex Lie algebra $\mathfrak{g}$. Here we compute Belavin-Drinfeld cohomology for all non-skewsymmetric $r$-matrices on the Belavin-Drinfeld list for simple Lie algebras of type $B, C$, and D. Published by AIP Publishing. [http://dx.doi.org/10.1063/1.4950895]
\end{abstract}

\section{INTRODUCTION}

The aim of the present paper is to classify quantum groups which have the universal enveloping algebra $U(\mathfrak{g})$ as their classical limit. In other words, we classify Hopf algebras $H$ over $\mathbb{C}[[\hbar]]$ such that $H / \hbar H$ is isomorphic to $U(\mathfrak{g})$ (with the standard cocommutative comultiplication) as Hopf algebras.

In our paper, $\mathfrak{g}$ is a simple finite dimensional Lie algebra of the series $B, C$, and $D$.

The first quantum group, whose classical limit is $U\left(s l_{2}\right)$, was discovered by Kulish and Reshetikhin in Ref. 9. Later, Drinfeld and Jimbo independently constructed quantization of $U(\mathfrak{g})$ for any simple Lie algebra $\mathfrak{g}$, see Refs. 2 and 7. Further examples of quantum groups were discovered by Etingof, Schedler, and Schiffmann in Ref. 6.

It follows from the results of our paper that the quantum groups above are governed by the so-called Belavin-Drinfeld cohomology.

Furthermore, our results show that the lists of the quantum groups above are incomplete. It turns out that there exists yet unknown class of quantum groups governed by the so-called twisted Belavin-Drinfeld cohomology.

Another intriguing discovery of our paper is that the Belavin-Drinfeld cohomology of the algebras of type $D_{2 n+1}$ is substantially different from other types of the classical Lie algebras.

We hope that our results, like the classification of simple Lie algebras, will find many applications in mathematics and mathematical physics, in first turn in the theory of integrable models.

\section{PRELIMINARIES}

In the papers ${ }^{3,4}$ Kazhdan and Etingof constructed the equivalence between the categories of quantum groups and Lie bialgebras over $\mathbb{C}[[\hbar]]$. The precise statement is in the following theorem.

Theorem 2.1. Let Qgroup be the category of quantum groups, i.e., topologically free cocommutative mod $\hbar$ Hopf algebras over $\mathbb{C}[[\hbar]]$ such that $H / \hbar H$ is a universal enveloping algebra of some Lie algebra $\mathfrak{g}$ over $\mathbb{C}$. Let LieBialg be the category of topologically free Lie bialgebras over $\mathbb{C}[[\hbar]]$ with $\delta=0$ mod $\hbar$. Then there exists a functor Lie : Qgroup $\rightarrow$ LieBialg that is an equivalence of categories.

This theorem can be seen as a quantization of the classical Lie theorem on the equivalence between the categories of simply connected Lie groups and Lie algebras. Lie theorem reduces the 
problem of classification for (say, semisimple) Lie groups to the same problem for Lie algebras that is much more easy. The same approach works in the quantum group case.

Our goal is to classify quantum groups whose quasiclassical limit is a given semisimple Lie algebra $\mathfrak{g}$. In order to do this we will classify Lie bialgebra structures on $\mathfrak{g}(\mathbb{C}[[\hbar]])$. From Theorem 2.1 we see that this classification will indeed be a classification of quantum groups.

Any Lie bialgebra structure on $\mathfrak{g}(\mathbb{C}[[\hbar]])$ can be lifted to a Lie bialgebra structure on $\mathfrak{g}(\mathbb{C}((\hbar)))$. Also any Lie bialgebra structure on $\mathfrak{g}(\mathbb{C}((\hbar)))$ defines a Lie bialgebra structure on $\mathfrak{g}(\mathbb{C}[[\hbar]])$ after multiplication by an appropriate power of $\hbar$. So we are left with the problem of classification of Lie bialgebra structures on $\mathfrak{g}(\mathbb{C}((\hbar)))$.

Summarizing the discussion above we have obtained the following: the classification of quantum groups is reduced to the classification of Lie bialgebras over $\mathbb{C}((\hbar))$.

According to Ref. 8, Lie bialgebra structures over $\mathbb{C}((\hbar))$ fall into three classes. Two of them are related to the Belavin-Drinfeld list of Ref. 1 and the third one is related to quasi-Frobenius subalgebras of $\mathfrak{g}(\mathbb{C}((\hbar)))$. It is known that the third class is wild, e.g., because it contains the classification problem of commutative subalgebras of $\mathfrak{g}$ of even dimension. In the present paper, we will classify the first two classes.

Recall the Belavin-Drinfeld classification. Let $\mathfrak{g}$ be a simple Lie algebra over an algebraically closed field of characteristic zero. Then any Lie bialgebra structure $\delta$ on $\mathfrak{g}$ is a coboundary one, i.e., is of the form $\delta(x)=[r, x \otimes 1+1 \otimes x]$, where $r$ is the corresponding $r$-matrix. The Belavin-Drinfeld theorem deals with the case $r$ is not skew-symmetric, i.e., $\delta$ is not triangular.

Let $\Gamma_{1}, \Gamma_{2}$ be subsets of the set of simple roots of $\mathfrak{g}, \tau: \Gamma_{1} \rightarrow \Gamma_{2}$ an isometric bijection such that for every $\alpha \in \Gamma_{1}$ there exists $k \in \mathbb{N}$ such that $\tau^{k}(\alpha) \in \Gamma_{1} \backslash \Gamma_{2}$. The triple $\left(\Gamma_{1}, \Gamma_{2}, \tau\right)$ is called admissible.

Let $K$ be the Killing form on $\mathfrak{g}$. For any root $\alpha$ choose $e_{\alpha} \in \mathfrak{g}_{\alpha}$ such that $K\left(e_{\alpha}, e_{-\alpha}\right)=1$.

Theorem 2.2 (Refs. 1 and 5). Let $r$ be an $r$-matrix on $g$ which is not skew-symmetric. Then for some root decomposition $r$ is a scalar multiple of

$$
r_{0}+\sum_{\alpha>0} e_{-\alpha} \otimes e_{\alpha}+\sum_{\alpha \in \operatorname{Span}\left(\Gamma_{1}\right)^{+}} \sum_{k \in \mathbb{N}} e_{-\alpha} \wedge e_{\tau^{k}(\alpha)},
$$

where $\left(\Gamma_{1}, \Gamma_{2}, \tau\right)$ is an admissible triple, and the tensor $r_{0} \in \mathfrak{h} \otimes \mathfrak{h}$ satisfies the following two conditions:

(1) $r_{0}+r_{0}^{21}=\sum t_{k} \otimes t_{k}$, where $t_{k}$ is an orthonormal basis of $\mathfrak{h}$ with respect to $K$,

(2) for any $\alpha \in \Gamma_{1}$ we have $(\tau(\alpha) \otimes \mathrm{id}+\mathrm{id} \otimes \alpha) r_{0}=0$.

Our goal is to obtain a version of this theorem for other fields of characteristic zero, possibly not algebraically closed.

\section{A. Setting}

In many cases we would prove the results for $B, C$, and $D$ series simultaneously. For this we will introduce some general notation and then specify it for each case. Note that further in the text we would also make distinction between $D_{n}$ algebras for odd and even $n$.

By $\mathfrak{g}(F)$ we denote the split simple Lie algebra of rank $n$ over a field $F$ of characteristic zero. We will work with Lie algebras of the $B, C, D$ series. By $G$ we denote a corresponding connected algebraic group, by $H$ a Cartan subgroup of $G$. The simple roots of $\mathfrak{g}$ are denoted $\alpha_{1}, \ldots, \alpha_{n}$. Let $e^{\alpha}$ be the character of $H$ that corresponds to the root $\alpha$. In all cases we will specify the isomorphism of $H$ with the standard torus $\left(F^{*}\right)^{n}$. Abusing notation, by $\left(d_{1}, \ldots, d_{n}\right)$ we denote the element of $H$ that corresponds to an element $\left(d_{1}, \ldots, d_{n}\right) \in\left(F^{*}\right)^{n}$. We will now specify the choice of matrix representation and root decomposition for the series $B, C, D$. By $M$ we will denote the size of matrices in each matrix.

\section{1. $B_{n}$ series}

Let $G$ be the subgroup of $S L(2 n+1)$ consisting of matrices satisfying $X B X^{T}=B$ (we have $M=2 n+1)$. Here $B$ is the matrix with 1's on the anti-diagonal and zeros elsewhere. Then $\mathfrak{g}$ is 
the algebra of matrices satisfying $X B+B X^{T}=0$. The Cartan subgroup $H$ (Cartan subalgebra, respectively) can be chosen to be the subset of diagonal matrices of $S L(2 n+1)$ (respectively, $\mathfrak{s l}(2 n+1))$. Simple root vectors are given by $e_{\alpha_{i}}=e_{i, i+1}-e_{2 n+2-i, 2 n+1-i}$. The Cartan subgroup is the subgroup of diagonal matrices of the form $\operatorname{diag}\left(d_{1}, \ldots, d_{n}, 1, d_{n}^{-1}, \ldots, d_{1}^{-1}\right)=:\left(d_{1}, \ldots, d_{n}\right)$. We have $e^{\alpha_{i}}\left(d_{1}, \ldots, d_{n}\right)=d_{i} d_{i+1}^{-1}$ for $i<n$ and $e^{\alpha_{n}}\left(d_{1}, \ldots, d_{n}\right)=d_{n}$.

\section{2. $C_{n}$ series}

Let $G$ be the subgroup of $S L(2 n)$ consisting of matrices satisfying $X B X^{T}=B$ (we have $M=2 n$ ). Here $B$ is the matrix with 1's on the upper half of the anti-diagonal, -1 's on the lower, and zeros elsewhere. E.g., for $n=2$

$$
B=\left(\begin{array}{cccc}
0 & 0 & 0 & 1 \\
0 & 0 & 1 & 0 \\
0 & -1 & 0 & 0 \\
-1 & 0 & 0 & 0
\end{array}\right)
$$

Then $\mathfrak{g}$ is the algebra of matrices satisfying $X B+B X^{T}=0$. The Cartan subgroup $H$ (Cartan subalgebra, respectively) can be chosen to be the subset of diagonal matrices of $S L(2 n)$ (respectively, $\mathfrak{s l}(2 n))$. Simple root vectors are given by $e_{\alpha_{i}}=e_{i, i+1}-e_{2 n+2-i, 2 n+1-i}$ for $i<n$ and $e_{\alpha_{n}}=e_{n, n+1}-$ $e_{n+1, n}$. The Cartan subgroup is the subgroup of diagonal matrices of the form $\operatorname{diag}\left(d_{1}, \ldots, d_{n}, d_{n}^{-1}, \ldots\right.$, $\left.d_{1}^{-1}\right)=:\left(d_{1}, \ldots, d_{n}\right)$. We have $e^{\alpha_{i}}\left(d_{1}, \ldots, d_{n}\right)=d_{i} d_{i+1}^{-1}$ for $i<n$ and $e^{\alpha_{n}}=d_{n}^{2}$.

\section{3. $D_{n}$ series}

Let $G$ be the subgroup of $S L(2 n)$ consisting of matrices satisfying $X B X^{T}=B$ (we have $M=$ $2 n$ ). Here $B$ is the matrix with 1's on the anti-diagonal and zeros elsewhere. Then $g$ is the algebra of matrices satisfying $X B+B X^{T}=0$. The Cartan subgroup $H$ (Cartan subalgebra, respectively) can be chosen to be the subset of diagonal matrices of $S L(2 n)$ (respectively, $\mathfrak{s l}(2 n)$ ). Simple root vectors are given by $e_{\alpha_{i}}=e_{i, i+1}-e_{2 n+2-i, 2 n+1-i}$ for $i<n$ and $e_{\alpha_{n}}=e_{n-1, n+1}-e_{n+2, n}$. The Cartan subgroup is the subgroup of diagonal matrices of the form $\operatorname{diag}\left(d_{1}, \ldots, d_{n}, d_{n}^{-1}, \ldots, d_{1}^{-1}\right)=:\left(d_{1}, \ldots, d_{n}\right)$. We have $e^{\alpha_{i}}\left(d_{1}, \ldots, d_{n}\right)=d_{i} d_{i+1}^{-1}$ for $i<n$ and $e^{\alpha_{n}}=d_{n-1} d_{n}$.

\section{B. Properties of $r$-matrices}

Let $\Omega \in\left(S^{2} \mathfrak{g}\right)^{\mathfrak{g}}$ be the Casimir element that corresponds to the Killing form $K$ on $\mathfrak{g}$. Note that $\Omega$ is defined over the field of rationals.

Lemma 2.3. If $r_{1}, r_{2}$ are two non-skewsymmetric $r$-matrices, $r_{1}+r_{1}^{21}=a \Omega$ and $r_{2}=r_{1}+b \Omega$, then $b=0$ or $b=-a$.

Proof. Let $\mathrm{CYB}(r)=\left[r^{12}, r^{13}\right]+\left[r^{12}, r^{23}\right]+\left[r^{13}, r^{23}\right]$ be the classical Yang-Baxter operator. Since $\operatorname{CYB}\left(r_{1}\right)=0$, we have $0=\operatorname{CYB}\left(r_{2}\right)=\operatorname{CYB}\left(r_{1}+b \Omega\right)=b(a+b)\left[\Omega^{12}, \Omega^{23}\right]$. Therefore $b=0$ or $b=-a$.

We will frequently work with the following objects.

Definition 2.4. Let $\left(\Gamma_{1}, \Gamma_{2}, \tau\right)$ be an admissible triple. Then a set of the form $\alpha, \tau(\alpha), \ldots, \tau^{k}(\alpha)$, where $\alpha \notin \Gamma_{2}$ and $\tau^{k}(\alpha) \notin \Gamma_{1}$ is called a string of $\tau$.

Definition 2.5. The centralizer $C(r)$ of an $r$-matrix $r$ is the set of all $X \in G(\bar{F})$ such that $\operatorname{Ad}_{X} r=r$.

The centralizer of a matrix on the Belavin-Drinfeld list can be explicitly described. 
Theorem 2.6 (Ref. 8). For any simple Lie algebra $\mathfrak{g}$ and for any Belavin-Drinfeld matrix $r_{B D}$ we have $C\left(r_{B D}\right) \subset H$. If $\left(\Gamma_{1}, \Gamma_{2}, \tau\right)$ is the admissible triple corresponding to $r_{B D}$, then $X \in C\left(r_{B D}\right)$ iff for any root $\alpha \in \Gamma_{1} \backslash \Gamma_{2}$ and for any $k \in \mathbb{N}$ we have $e^{\alpha}(X)=e^{\tau^{k}(\alpha)}(X)$, i.e., $e^{\alpha}(X)$ is constant on the strings of $\tau$.

Theorem 2.7. Let $r$ be a non-skewsymmetric $r$-matrix that defines a Lie bialgebra structure on $\mathfrak{g}(F), r+r^{21}=a \Omega$, where $a \in \bar{F}$. Then $a^{2} \in F$.

Proof. Recall that a Lie bialgebra structure $\delta$ corresponding to $r$ is given by the formula $\delta(x)=[r, x \otimes 1+1 \otimes x]$. Take an arbitrary $\sigma \in \operatorname{Gal}(\bar{F} / F)$. Consider the $r$-matrix $\sigma(r)$. As $r$ defines a Lie bialgebra structure on $\mathfrak{g}(F)$, we have $\delta(x)=\sigma(\delta(x))$ for all $x \in \mathfrak{g}(F)$. In particular, $\sigma(r)-r \in(\mathfrak{g} \otimes \mathfrak{g})^{\mathfrak{g}}=\left(S^{2} \mathfrak{g}\right)^{\mathfrak{g}}$. Therefore $\sigma(r)=r+b \Omega$. From Lemma 2.3 we get $b=0$ or $b=-a$. On the other hand $\sigma(a) \Omega=\sigma(r)+\sigma\left(r^{21}\right)=r+r^{21}+2 b \Omega=(a+2 b) \Omega= \pm a \Omega$. Therefore $\sigma(a)=$ $\pm a$. Thus $\sigma\left(a^{2}\right)=a^{2}$ for all $\sigma \in \operatorname{Gal}(\bar{F} / F)$, i.e., $a^{2} \in F$.

\section{Matrix lemmas}

Lemma 2.8. If for some $X \in G L(n+m, \bar{F})$ and for any $\sigma \in \operatorname{Gal}(\bar{F} / F)$ the matrix $X^{-1} \sigma(X)$ is a $n \times n, m \times m$ block matrix, then there exists $Q \in G L(n+m, F)$ and a block matrix $K$ such that $X=Q K$.

Proof. Let $X=\left(\begin{array}{ll}A & B \\ C & D\end{array}\right)$. Rearranging rows if necessary, we can assume that $A$ and $D$ are nondegenerate (because of Laplace formula). Then $\sigma(X)=X K$, where $K=\left(\begin{array}{cc}K_{1} & 0 \\ 0 & K_{2}\end{array}\right) \in G L(n+m, \bar{F})$. Then we have $\sigma(A)=A K_{1}$ and $\sigma(C)=C K_{1}$. Since $C A^{-1}$ is $\operatorname{Gal}(\bar{F} / F)$-stable we have $C=F_{1} A$, $F_{1} \in G L(m \times n, F)$. Similarly $B=F_{2} D, F_{2} \in G L(n \times m, F)$. Finally $\left(\begin{array}{cc}A & B \\ C & D\end{array}\right)=\left(\begin{array}{cc}I & F_{2} \\ F_{1} & I\end{array}\right)\left(\begin{array}{cc}A & 0 \\ 0 & D\end{array}\right)$.

Corollary 2.9. If for some $X \in G L(n, F)$ and for any $\sigma \in \operatorname{Gal}(\bar{F} / F)$ the matrix $X^{-1} \sigma(X)$ is diagonal, then $X=Q D$ where $D$ is diagonal and $Q \in G L(n, F)$.

\section{OVERVIEW OF THE PAPER}

Our main goal is to classify Lie bialgebra structures on $\mathfrak{g}(F)$. We will mostly be interested in the case $F=\mathbb{C}((\hbar))$; however, many general results hold for an arbitrary field of characteristic zero. Because any Lie bialgebra structure on $\mathfrak{g}(F)$ extends to a Lie bialgebra structure on $\mathfrak{g}(\bar{F})$, we have

Theorem 3.1. Any Lie bialgebra structure on $\mathrm{g}(F)$ is a coboundary one given by an r-matrix. If $r$ is not skewsymmetric, then it has the form $r=a \operatorname{Ad}_{X} r_{B D}$, where $a \in \bar{F}^{*}, X \in G(\bar{F}), r_{B D}$ is an $r$-matrix on the Belavin-Drinfeld list.

However, not all $r$-matrices of the form $r=a \operatorname{Ad}_{X} r_{B D}$ define a Lie bialgebra structure on $\mathrm{g}(F)$.

In what follows we will be interested only in non-skewsymmetric $r$-matrices. We will classify all (non-skewsymmetic) $r$-matrices that do define a Lie bialgebra structure on $\mathfrak{g}(F)$ up to the following equivalence.

Definition 3.2. Two $r$-matrices $r_{1}$ and $r_{2}$ are called equivalent if for some $a \in F^{*}$ and $X \in G(F)$ we have $r_{1}=a \operatorname{Ad}_{X} r_{2}$.

By the Belavin-Drinfeld classification every $r$-matrix is equivalent to $r_{B D}$ over $\bar{F}$, and $r_{B D}$ 's for different triples $\left(\Gamma_{1}, \Gamma_{2}, \tau\right)$ are not equivalent. However, over $F$ each $\bar{F}$-equivalence class can split into several equivalence classes. It turns out that if $a \mathrm{Ad}_{X} r_{B D}$ defines a Lie bialgebra structure on $\mathfrak{g}(F)$ then $a^{2} \in F$. Therefore we have a class of $r$-matrices for each $a^{2} \in F^{*} /\left(F^{*}\right)^{2}$. Elements from different classes are not equivalent, but each class can further split into several equivalence classes. For $F=\mathbb{C}((\hbar))$ we have only two elements in $F^{*} /\left(F^{*}\right)^{2}$ : the class of 1 and the class of $\hbar$. 
For every element $u \in F^{*} /\left(F^{*}\right)^{2}$ we will introduce the Belavin-Drinfeld cohomologies that parameterize equivalence classes of $r$-matrices of the form $a \operatorname{Ad}_{X} r_{B D}$, where $a^{2}=u$ in $F^{*} /\left(F^{*}\right)^{2}$. The cohomologies are called twisted if $u \neq 1$ and non-twisted otherwise.

Henceforth we will assume that $r_{B D}$ is defined over $F$, i.e., $r_{0} \in \mathfrak{g}(F) \otimes \mathfrak{g}(F)$.

\section{A. Non-twisted cohomologies}

The following theorem holds:

Theorem 3.3 (Ref. 8). Ad $d_{X} r_{B D}$ defines a Lie bialgebra structure on $\mathrm{g}(F)$ if and only if for any $\sigma \in \operatorname{Gal}(\bar{F} / F)$ we have $X^{-1} \sigma(X) \in C(r)$.

With this theorem in mind we can define Belavin-Drinfeld cohomologies without referring to Lie bialgebra structures.

Definition 3.4. $X \in G(\bar{F})$ is called a non-twisted Belavin-Drinfeld cocycle for $r_{B D}$ if for any $\sigma \in \operatorname{Gal}(\bar{F} / F)$ we have $X^{-1} \sigma(X) \in C\left(r_{B D}\right)$. The set of non-twisted cocycles will be denoted $Z\left(r_{B D}\right)=Z\left(G, r_{B D}\right)$.

In other words $X \in Z\left(r_{B D}\right)$ if $\operatorname{Ad}_{X} r_{B D}$ defines a Lie bialgebra structure on $\mathfrak{g}(F)$.

Definition 3.5. Two cocycles $X_{1}, X_{2} \in Z\left(r_{B D}\right)$ are called equivalent if there exist $Q \in G(F)$ and $C \in C\left(r_{B D}\right)$ such that $X_{1}=Q X_{2} C$.

It is easy to see that $X_{1}$ and $X_{2}$ are equivalent if and only if $\operatorname{Ad}_{X_{1}} r_{B D}$ and $\operatorname{Ad}_{X_{2}} r_{B D}$ are equivalent as $r$-matrices.

Definition 3.6. The set of equivalence classes of non-twisted cocycles is denoted $H_{B D}^{1}\left(r_{B D}\right)=$ $H_{B D}^{1}\left(G, r_{B D}\right)$ and is called non-twisted Belavin-Drinfeld cohomologies.

Remark 3.7. The equivalent way to treat Belavin-Drinfeld cohomology is to understand it as a Galois cohomology of the Galois module $C(r)$ (over the absolute Galois group of $F$ ). This justifies the use of the word "cohomology." Also this approach enables us to put a natural group structure on $H_{B D}^{1}(r)$. Indeed, for any $X \in Z(r)$ the map $\operatorname{Gal}(\bar{F} / F) \rightarrow C(r)$ given by $\sigma \mapsto X^{-1} \sigma(X)$ is a 1-cocycle. If such a map is equivalent to the identity then there exists $C \in C(r)$ such that $\left(X C^{-1}\right)^{-1} \sigma\left(X C^{-1}\right)=1$ for all $\sigma$. Then one can find $Q \in G(F)$ such that $X C^{-1}=Q$. This means that our equivalence relation is the same as the equivalence of Galois cocycles. We also need to prove that any Galois cocycle is of the given form. We do not know how to prove this in general, however for the algebras of $B, C, D$ types we sketch a proof.

First observe that by Hilbert's theorem 90 it is always possible to find $Y \in G L(n, \bar{F})$ such that $Y^{-1} \sigma(Y)=X^{-1} \sigma(X) \in C(r)$ for all $\sigma \in \operatorname{Gal}(\bar{F} / F)$. With the suitable matrix representation of the group $G$ we can assume that $C(r)$ is a subset of the set of diagonal matrices. Then by Lemma 2.8 we can assume that $Y$ is diagonal. By looking at the equations that define $H$ inside the set of diagonal matrices we can decompose $Y$ as $Y=Q Y^{\prime}$ with $Y^{\prime} \in C(r)$ and $Q \in \operatorname{diag}(n, F)$. Then $Y^{\prime}$ is in $C(r)$ and gives rise to the same cocycle as $Y$.

Computation of $H_{B D}^{1}\left(G, r_{B D}\right)$ is possible because of the easy description of $C\left(r_{B D}\right)$ (see Theorem 2.6).

\section{B. Twisted cohomologies}

Twisted case is somewhat similar to the non-twisted one, however computations become more complicated. Fix a non-zero $a \in \bar{F}$ such that $a^{2} \in F$. We will mostly work with $F=\mathbb{C}((\hbar))$ and $a=\sqrt{\hbar}$. We have the following theorem.

Theorem 3.8 (Ref. 8). $a \operatorname{Ad}_{X} r_{B D}$ defines a Lie bialgebra structure on $\mathrm{g}(F)$ if and only if $X$ is a non-twisted cocycle for the field $F[a]$ and $\operatorname{Ad}_{X^{-1} \sigma_{0}(X)} r_{B D}=r_{B D}^{21}$. Here $\sigma_{0}$ is the nontrivial element of $\operatorname{Gal}(F[a] / F)$. 
To deal with the condition $\operatorname{Ad}_{X^{-1} \sigma_{0}(X)} r_{B D}=r_{B D}^{21}$ we will classify all triples $\left(\Gamma_{1}, \Gamma_{2}, \tau\right)$ such that $r_{B D}^{21}$ and $r_{B D}$ are conjugate. In each case we will find suitable $S \in G(F)$ such that $r_{B D}^{21}=\operatorname{Ad}_{S} r_{B D}$. Then we can define Belavin-Drinfeld cocycles and cohomologies similar to the non-twisted case. In all cases $S^{2}= \pm 1$.

Definition 3.9. $X \in G(\bar{F})$ is called a Belavin-Drinfeld twisted cocycle if for any $\sigma \in \operatorname{Gal}(\bar{F} /$ $F[a])$ we have $X^{-1} \sigma(X) \in C\left(r_{B D}\right)$ and $S X^{-1} \sigma_{0}(X) \in C\left(r_{B D}\right)$. The set of Belavin-Drinfeld twisted cocycles is denoted $\bar{Z}\left(r_{B D}\right)=\bar{Z}\left(G, r_{B D}\right)$.

Definition 3.10. Two twisted cocycles $X_{1}, X_{2}$ are called equivalent if there exist $Q \in G(F)$ and $C \in C\left(r_{B D}\right)$ such that $X_{1}=Q X_{2} C$. The set of equivalence classes of twisted cocycles is called twisted Belavin-Drinfeld cohomologies and is denoted by $\bar{H}_{B D}^{1}\left(r_{B D}\right)=\bar{H}_{B D}^{1}\left(G, r_{B D}\right)$.

Proposition 3.11. Two r-matrices $a \mathrm{Ad}_{X} r_{B D}$ and $a \mathrm{Ad}_{Y} r_{B D}$ are conjugate by an element of $G(F)$ if and only if $X$ and $Y$ are equivalent as twisted cocycles. Thus the set of equivalence classes of twisted Lie bialgebra structures is $\bar{H}_{B D}^{1}(G, r)$.

To find $\bar{H}_{B D}^{1}\left(r_{B D}, G\right)$ we proceed as follows. First we find a matrix $J$ such that $J \in G L(F[a])$ and $\bar{J}=S J$ (where $\bar{T}:=\sigma_{0}(T)$ ). Then we will show that all Belavin-Drinfeld cocycles are of the form $R J D$ with $R \in G L(F)$ and $D$ diagonal. We will then show that for a diagonal matrix $D$ that satisfies some additional assumptions we can find $R$ such that $R J D$ is a cocycle. We will also see that the equivalence of $R_{1} J D_{1}$ and $R_{2} J D_{2}$ can be understood purely in terms of $D_{1}$ and $D_{2}$. Therefore the problem reduces to classification of classes of certain diagonal matrices under some equivalence. This will be done explicitly.

\section{Results}

We summarize the results of the paper in Tables I and II.

\section{NON-TWISTED COHOMOLOGIES}

Lemma 4.1. The Belavin-Drinfeld cohomology associated to the Drinfeld-Jimbo r-matrix is trivial.

Proof. Let $X \in Z\left(r_{D J}\right)$. By Theorem 2.6 and Corollary 2.9 we have $X=Q D$ where $Q \in$ $G L(M, F)$ and $D \in \operatorname{diag}(M, \bar{F})$. Hence, for any $\sigma \in \operatorname{Gal}(\bar{F} / F)$ we have $D^{-1} \sigma(D) \in C\left(r_{D J}\right) \subset H$. This implies $d_{i} d_{M+1-i}=k_{i} \in F$ (for $B_{n}$ series we also have $d_{[(M+1) / 2]} \in F$ ). Let $K=\operatorname{diag}\left(k_{1}, \ldots\right.$, $\left.k_{[M / 2]}, 1,1, \ldots, 1\right)$ (for $B_{n}$ let $K=\operatorname{diag}\left(k_{1}, \ldots, k_{[M / 2]}, d_{[(M+1) / 2]}, 1, \ldots, 1\right)$ ). Then $D_{1}=K^{-1} D \in G$. We have $X=(Q K) \cdot D_{1}$. Thus $X \sim I$.

Remark 4.2. It is easy to see that $C\left(r_{D J}\right)=H$. Therefore for an arbitrary $r$-matrix $r$ we have $Z(r) \subset Z\left(r_{D J}\right)$.

TABLE I. Non-twisted Belavin-Drinfeld cohomologies.

\begin{tabular}{llcc}
\hline \hline Algebra & Triple type & $H_{B D}^{1}$ for an arbitrary field & $H_{B D}^{1}$ for $\mathbb{C}((\hbar))$ \\
\hline$B_{n}$ & & Trivial & \\
\hline$C_{n}$ & There exists a string of $\tau$ that contains $\alpha_{n-1}$ and $\alpha_{n}$ & $F^{*} /\left(F^{*}\right)^{2}$ & 2 elements \\
\hline$n_{n}$ & $\alpha_{n-1}$ and $\alpha_{n}$ do not belong to the same string of $\tau$ & Trivial & \\
\hline \hline
\end{tabular}


TABLE II. Twisted Belavin-Drinfeld cohomologies.

\begin{tabular}{|c|c|c|c|}
\hline Algebra & & Triple type & $\bar{H}_{B D}^{1}$ for $\mathbb{C}((\hbar))$ \\
\hline \multirow{2}{*}{$B_{n}$} & & Drinfeld-Jimbo & One element \\
\hline & & not DJ & empty \\
\hline \multirow{2}{*}{$C_{n}$} & & Drinfeld-Jimbo & One element \\
\hline & & not DJ & empty \\
\hline \multirow{5}{*}{$D_{n}$} & \multirow{2}{*}{ Even $n$} & Drinfeld-Jimbo & One element \\
\hline & & not DJ & empty \\
\hline & \multirow[t]{3}{*}{ Odd $n$} & $\begin{array}{l}\Gamma_{1}=\left\{\alpha_{n-1}\right\} \\
\tau\left(\alpha_{n-1}\right)=\alpha_{n} ; \\
\Gamma_{1}=\left\{\alpha_{n}\right\} \\
\tau\left(\alpha_{n}\right)=\alpha_{n-1} ; \\
\Gamma_{1}=\left\{\alpha_{n-1}, \alpha_{k}\right\}, k \neq n \\
\tau\left(\alpha_{n-1}\right)=\alpha_{k}, \tau\left(\alpha_{k}\right)=\alpha_{n} ; \\
\Gamma_{1}=\left\{\alpha_{n}, \alpha_{k}\right\}, k \neq n-1 \\
\tau\left(\alpha_{n}\right)=\alpha_{k}, \tau\left(\alpha_{k}\right)=\alpha_{n-1} \\
\end{array}$ & Two elements \\
\hline & & Drinfeld-Jimbo & One element \\
\hline & & other $r_{B D}$ & empty \\
\hline
\end{tabular}

Let $r$ be an $r$-matrix from the Belavin-Drinfeld list associated to a triple $\left(\Gamma_{1}, \Gamma_{2}, \tau\right)$.

Theorem 4.3. For any $r$-matrix in the $B_{n}, C_{n}$ series $H_{B D}^{1}(r)$ contains one element. For the $D_{n}$ series if there exists $k \in \mathbb{Z}$ such that $\tau^{k}\left(\alpha_{n-1}\right)=\alpha_{n}$, then $H_{B D}^{1}(r)$ is parameterized by the elements of $F^{*} /\left(F^{*}\right)^{2}$. Otherwise $H_{B D}^{1}(r)$ is trivial.

Proof. First note that in the $B_{n}$ and $C_{n}$ series there is no $k$ such that $\tau^{k}\left(\alpha_{n-1}\right)=\alpha_{n}$, because $\alpha_{n-1}$ and $\alpha_{n}$ have different length.

Consider a homomorphism $T: H(\bar{F}) \rightarrow \operatorname{diag}(n, \bar{F}) \subset G L(n, \bar{F})$ defined by $T\left(d_{1}, \ldots, d_{n}\right)=\left(s_{1}\right.$, $\left.\ldots, s_{n}\right), s_{i}=e^{\alpha_{i}}\left(d_{1}, \ldots, d_{n}\right)$. Then $T$ is surjective and its kernel is contained in the subset of $\left(d_{1}, \ldots, d_{n}\right) \in H$ such that $d_{1}=\cdots=d_{n}$. From the explicit description of the root characters we see that for $s_{1}, \ldots, s_{n} \in F$ we can find an element of $H(F)$ in $T^{-1}\left(s_{1}, \ldots, s_{n}\right)$ if and only if the following holds: for the $D_{n}$ case $s_{n-1} s_{n}$ is a perfect square, for the $C_{n}$ case $s_{n}$ is a perfect square, and always for the $B_{n}$ series.

For a positive number $i$ such that $\alpha_{i} \in \Gamma_{1}$ let us consider the string of $\tau$ that contains $\alpha_{i}$. By $\eta(i)$ we will denote the smallest number $k$ such that $k>i$ and $\alpha_{k}$ lies in the same string as $\alpha_{i}$. If $\alpha_{i} \notin \Gamma_{1}$, then we define $\eta(i)=0$. For $D \in H$ let $s_{i}=e^{\alpha_{i}}(D)$.

For any $X \in Z\left(r_{B D}\right)$ we have a decomposition $X=Q D$. Let $D=\left(d_{1}, \ldots, d_{n}\right)$ by Lemma 4.1.

Case 1. $\eta(n-1)=0$. If $D=\left(d_{1}, \ldots, d_{n}\right)$ is a diagonal matrix then the tuple $\left(s_{1}, \ldots, s_{n-1}\right)$ defines $D$ up to a scalar. $D \in C\left(r_{B D}\right)$ iff $s_{i}$ 's are equal on the strings of $\tau$. We have $D^{-1} \sigma(D) \in$ $C\left(r_{B D}\right)$. This means that for all $\alpha_{i}, \alpha_{j}$ such that $\tau^{k}\left(\alpha_{i}\right)=\alpha_{j}$ we have $s_{i}=k_{i} s_{j}, k_{i} \in F$. We can find a matrix $D^{\prime}=\left(d_{1}^{\prime}, \ldots, d_{n}^{\prime}\right) \in H(F)$ for which $s_{i}^{\prime}=k_{i} s_{j}^{\prime}$ for all $i, j$ such that $\tau^{k}\left(\alpha_{i}\right)=\alpha_{j}$ for some $k$. Then the decomposition $X=\left(Q D^{\prime}\right) \cdot I \cdot\left(D^{\prime-1} D\right)$ gives an equivalence between $X$ and $I$.

Case 2. $\eta(n-1)=n$ (possible only in the $D_{n}$ algebra). Again we have $s_{i}=k_{i} s_{\eta(i)}$ for $k_{i} \in F$. The equations $s_{i}=k_{i} s_{\eta(i)}$ for $i=1, \ldots, n-1$ and $s_{n-1}=k_{n-1}^{2} s_{n}$ have a solution $d_{1}^{\prime}, \ldots, d_{n}^{\prime} \in F$. Let $I^{\prime}=\left(1, \ldots, 1, \sqrt{k_{n-1}}\right)$. Then $X=\left(Q D^{\prime}\right) I^{\prime}\left(D^{\prime-1} D\right)$. Therefore, every cocycle is equivalent to one of the form $(1, \ldots, 1, \sqrt{k})$ for some $k \in F$. Let $I^{\prime}=(1, \ldots, 1, \sqrt{k})$ and $I^{\prime \prime}=(1, \ldots, 1, \sqrt{l})$. If $I^{\prime \prime} \sim I^{\prime}$, then $I^{\prime}=Q I^{\prime \prime} C$, where $Q \in H(F)$ and $C \in C\left(r_{B D}\right)$. But then $k / l$ must be a square in $F$. Therefore, $H_{B D}^{1}(r)$ is parameterized by the elements of $F^{*} /\left(F^{*}\right)^{2}$. 


\section{TWISTED COHOMOLOGIES}

In this section we work over the field $\mathbb{K}=\mathbb{C}((\hbar))$.

From now on we will make distinction not only between $B, C, D$ series but also between the algebras from $D_{n}$ series for odd and even $n$. By $S$ we denote the following matrix:

the matrix with 1's on the antidiagonal for the $D_{n}, n$ even, and $B_{n}$ algebras;

the matrix with 1's on the antidiagonal but with central $2 \times 2$ block replaced with a unit matrix for $D_{n}$ with odd $n$;

the matrix with -1 's on the upper half of the antidiagonal and 1's on the lower half for the $C_{n}$ algebra.

Example 5.1. The matrices $S$ for the algebras $D_{2}, D_{3}, B_{2}$, and $C_{2}$, respectively, are

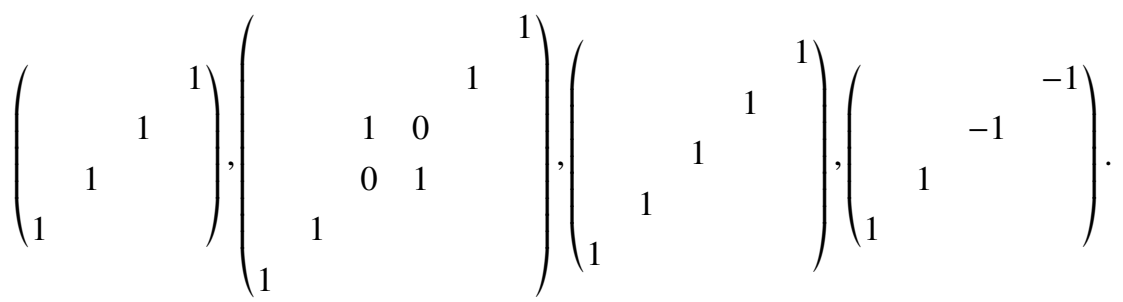

Note that we always have $S \in G$ and $S^{2}= \pm$ id with $S^{2}=-$ id only for $C_{n}$ algebra.

Recall that if $\bar{H}_{B D}^{1}(G, r)$ is nonempty, then $r^{21}$ and $r$ are conjugate.

Lemma 5.2. If $r$ corresponds to a triple $\left(\Gamma_{1}, \Gamma_{2}, \tau\right)$ and $r$ and $r^{21}$ are conjugate, then $r^{21}$ and $\mathrm{Ad}_{S} r$ are conjugate by an element of $C\left(r_{D J}\right)$.

Proof. It is easy to see that $\operatorname{Ad}_{S} e_{\alpha}=e_{-\alpha}$ for all cases except $D_{n}$ with odd $n$. For $D_{n}$ with odd $n$ we have $\operatorname{Ad}_{S} e_{\alpha_{i}}=e_{-\alpha_{i}}$ for $i=1, \ldots, n-2$ and $\operatorname{Ad}_{S} e_{\alpha_{n}}=e_{-\alpha_{n-1}}, \operatorname{Ad}_{S} e_{\alpha_{n-1}}=e_{-\alpha_{n}}$. In all cases $\operatorname{Ad}_{S} r_{D J}=r_{D J}^{21}$. This means that $\operatorname{Ad}_{S} r$ and $r^{21}$ have the same semisimple parts. As they are conjugate we can conclude that they are conjugate by an element of $C\left(r_{D J}\right)$.

Lemma 5.3. If $r$ corresponds to a triple $\left(\Gamma_{1}, \Gamma_{2}, \tau\right)$ and $r$ and $r^{21}$ are conjugate, then the triple is one of the following:

for $B_{n}, C_{n}$, or $D_{n}$ with even $n: \Gamma_{1}=\Gamma_{2}=\emptyset$;

for $D_{n}$ with odd $n: \Gamma_{1}=\Gamma_{2}=\emptyset$ or one of the following cases:

1. $\Gamma_{1}=\left\{\alpha_{n-1}\right\}, \tau\left(\alpha_{n-1}\right)=\alpha_{n}$;

2. $\Gamma_{1}=\left\{\alpha_{n}\right\}, \tau\left(\alpha_{n}\right)=\alpha_{n-1}$;

3. $\Gamma_{1}=\left\{\alpha_{n-1}, \alpha_{k}\right\}, \tau\left(\alpha_{n-1}\right)=\alpha_{k}, \tau\left(\alpha_{k}\right)=\alpha_{n}$

4. $\Gamma_{1}=\left\{\alpha_{n}, \alpha_{k}\right\}, \tau\left(\alpha_{n}\right)=\alpha_{k}, \tau\left(\alpha_{k}\right)=\alpha_{n-1}$.

In these cases if $r_{0}^{21}=\operatorname{Ad}_{S} r_{0}$, then $r^{21}=\operatorname{Ad}_{S} r$.

Proof. First consider the series $B_{n}, C_{n}$, or $D_{n}$ with even $n$. Let $\alpha, \beta$ be simple roots. If $e_{-\alpha} \wedge e_{\beta}$ is a summand of $r$, then $\operatorname{Ad}_{S} r$ contains $e_{\alpha} \wedge e_{-\beta}$ and $r^{21}$ contains $e_{\beta} \wedge e_{-\alpha}$. Therefore, if $\alpha=\tau(\beta)$, then $\beta=\tau^{k}(\alpha)$ for some $k$, i.e., $\Gamma_{1}=\Gamma_{2}=\emptyset$.

For $D_{n}$ with odd $n$ the same works if $\alpha, \beta \notin\left\{\alpha_{n-1}, \alpha_{n}\right\}$. Since $\operatorname{Ad}_{S} e_{\alpha_{n-1}}=e_{-\alpha_{n}}, \operatorname{Ad}_{S} e_{\alpha_{n}}=$ $e_{-\alpha_{n-1}}$, we can also get triples of the form $1-4$.

On the other hand, it is straightforward to see that for each of the triples listed in the statement of the lemma we have $r^{21}=\operatorname{Ad}_{S} r$ provided that $r_{0}^{21}=\operatorname{Ad}_{S} r_{0}$.

Remark 5.4. Let $s \in$ Aut $\Gamma$ be the involutive automorphism of the Dynkin diagram induced by $S$. If an $r$-matrix $r$ corresponds to an admissible triple $\left(\Gamma_{1}, \Gamma_{2}, \tau\right)$, and $\bar{H}_{B D}^{1}(G, r)$ is nonempty, then $s\left(\Gamma_{1}\right)=\Gamma_{2}$ and $s \tau=\tau^{-1} s$. It is easy to show (cf., Ref. 10) that for any such triple $\left(\Gamma_{1}, \Gamma_{2}, \tau\right)$ there exists a tensor $r_{0} \in \mathfrak{h} \otimes \mathfrak{h}$ that satisfies the conditions of Theorem 2.2 and the condition $r_{0}^{21}=\operatorname{Ad}_{S} r_{0}$. Moreover, such tensors form an affine space of dimension $l(k-l)$, where $k=\left|\Gamma \backslash \Gamma_{1}\right|$ is the number 
of strings of $\tau$, and $l$ is the number of 2-element orbits of $s$ on the set of strings of $\tau$. Notice that this description is applicable also to the $A$ series (cf., Refs. 8 and 11).

In particular, $s=$ id for all Lie algebras of the $B, C, D$ series, except for $D_{n}$ with $n$ odd, where $s$ interchanges $\alpha_{n}$ and $\alpha_{n-1}$. Therefore, for $D_{n}$ with $n$ odd and $\Gamma_{1}=\Gamma_{2}=\emptyset$, tensors $r_{0}$ that satisfy the conditions of Theorem 2.2 and the condition $r_{0}^{21}=\mathrm{Ad}_{S} r_{0}$ form an affine space of dimension $n-1$. In all other cases listed in Lemma 5.3 such tensor $r_{0}$ is unique.

Note that the Belavin-Drinfeld cohomology is independent of the choice of $r_{0}$.

In the sequel we will assume that $r_{0}$ satisfies the condition $r_{0}^{21}=\mathrm{Ad}_{S} r_{0}$.

Remark 5.5. Assume that $X \in G(\overline{\mathbb{K}})$ is a Belavin-Drinfeld twisted cocycle associated to $r$. The identity $\left(\operatorname{Ad}_{X^{-1} \sigma_{0}(X)} \otimes \operatorname{Ad}_{X^{-1} \sigma_{0}(X)}\right)(r)=r^{21}$ is equivalent to $\left(\operatorname{Ad}_{S^{-1} X^{-1} \sigma_{0}(X)} \otimes \operatorname{Ad}_{S^{-1} X^{-1} \sigma_{0}(X)}\right)(r)=$ $r$, which implies that $S^{-1} X^{-1} \sigma_{0}(X) \in C(r)$. Thus $X^{-1} \sigma_{0}(X)=S D$ with $D \in C(r)$.

On the other hand, $\left(\operatorname{Ad}_{X^{-1} \sigma(X)} \otimes \operatorname{Ad}_{X^{-1} \sigma(X)}\right)(r)=r$ holds for any $\sigma \in \operatorname{Gal}(\overline{\mathbb{K}} / \mathbb{K}[\sqrt{\hbar}])$. This in turn is equivalent to $X^{-1} \sigma(X)=D_{\sigma} \in C(r)$.

We will now introduce a specific matrix $J \in G L(M, \mathbb{K}[\sqrt{\hbar}])$ for the $B, C, D$ series. In every case we will have $\bar{J}:=\sigma_{0}(J)=J S$. Again, notice the distinction between $D_{n}$ for odd and even $n$.

For $D_{n}, n$ even denote by $J=J\left(D_{n}\right) \in G L(2 n, \mathbb{K}[\sqrt{\hbar}])$ the matrix with the following entries: $a_{k k}=1$ for $k \leq n, a_{k k}=-\sqrt{\hbar}$ for $k \geq n+1, a_{k, 2 n+1-k}=1$ for $k \leq n, a_{k, 2 n+1-k}=\sqrt{\hbar}$ for $k \geq n+1$.

For $D_{n}, n$ odd let $J$ be a matrix $J\left(D_{n-1}\right)$ with the unit matrix inserted as the central $2 \times 2$ block.

For $B_{n}$ let $J$ be a matrix $J\left(D_{n}\right)$ with the unit matrix inserted as the central $1 \times 1$ block.

For $C_{n}$ take $J$ to be $J\left(D_{n}\right)$.

E.g., for $D_{2}$ and $C_{2}$ we have

$$
J=\left(\begin{array}{cccc}
1 & 0 & 0 & 1 \\
0 & 1 & 1 & 0 \\
0 & \sqrt{\hbar} & -\sqrt{\hbar} & 0 \\
\sqrt{\hbar} & 0 & 0 & -\sqrt{\hbar}
\end{array}\right),
$$

for $D_{3}$ we have

$$
J=\left(\begin{array}{cccccc}
1 & & & \cdots & & 1 \\
& 1 & & \cdots & 1 & \\
\cdots & & 1 & 0 & \cdots & \\
\cdots & & 0 & 1 & \cdots & \\
& \sqrt{\hbar} & & \ldots & -\sqrt{\hbar} & \\
\sqrt{\hbar} & & & \ldots & & -\sqrt{\hbar}
\end{array}\right),
$$

and for $B_{2}$ we have

$$
J=\left(\begin{array}{ccccc}
1 & 0 & 0 & 0 & 1 \\
0 & 1 & 0 & 1 & 0 \\
0 & 0 & 1 & 0 & 0 \\
0 & \sqrt{\hbar} & 0 & -\sqrt{\hbar} & 0 \\
\sqrt{\hbar} & 0 & 0 & 0 & -\sqrt{\hbar}
\end{array}\right) .
$$

Lemma 5.6. Let $X \in \bar{Z}(G, r)$. Then there exist $R \in G L(M, \mathbb{K})$ and $D \in \operatorname{diag}(M, \overline{\mathbb{K}})$ such that $X=R J D$. Moreover, $D=\operatorname{diag}\left(d_{1}, \ldots, d_{M}\right)$, where $d_{i} d_{M-i} \in \mathbb{K}$ for all $i$ (for $B_{n}$ series we also have $d_{n+1}=1$, for $C_{n}$ series we have $\left.d_{i} d_{M-i} \in \sqrt{\hbar} \mathbb{K}\right)$, and for any $\sigma \in \mathrm{Gal}(\overline{\mathbb{K}} / \mathbb{K}[\sqrt{\hbar}])$ we have $D^{-1} \sigma(D) \in C(r)$.

Proof. By Theorem 2.6 we have $C(r) \subset H$. Note that in the equation $\bar{X}=X S D$ the matrix $S D$ is a block matrix with $2 \times 2$ blocks. Applying Lemma 2.8 we see that $X=R X_{1}$ with $X_{1}$ having the same block form. If we would provide an appropriate decomposition for each $2 \times 2$ block we would 
obtain the desired decomposition. In each block the equation can be equivalent to the non-twisted cohomology equation (if in $S$ we have a unit block) or as one of the equations considered below. For $\sigma \in \operatorname{Gal}(\overline{\mathbb{K}} / \mathbb{K}[\sqrt{\hbar}])$ we have $R J D C=\sigma(R J D)=R J \sigma(D)$, where $C \in C(r)$.

Let $X=\left(\begin{array}{ll}a & b \\ c & d\end{array}\right) \in G L(2, \mathbb{K}[\sqrt{\hbar}])$ satisfy $\bar{X}=X W D$ with $D=\operatorname{diag}\left(d_{1}, d_{2}\right) \in S O(2, \mathbb{K}[\sqrt{\hbar}])$, where $W=\left(\begin{array}{ll}0 & 1 \\ 1 & 0\end{array}\right)$. The identity is equivalent to the following system: $\bar{a}=b d_{1}, \bar{b}=a d_{2}, \bar{c}=d d_{1}$, $\bar{d}=c d_{2}$. Note that $c d \neq 0$ (otherwise both $c$ and $d$ should be zero). Let $a / c=a^{\prime}+b^{\prime} \sqrt{\hbar}$. Then $b / d=a^{\prime}-b^{\prime} \sqrt{\hbar}$. One can immediately check that $X=R J^{\prime} D^{\prime}$, where $R=\left(\begin{array}{cc}a^{\prime} & b^{\prime} \\ 1 & 0\end{array}\right) \in G L(2, \mathbb{K})$, $J^{\prime}=\left(\begin{array}{cc}1 & 1 \\ \sqrt{\hbar} & -\sqrt{\hbar}\end{array}\right), D^{\prime}=\operatorname{diag}(c, d) \in \operatorname{diag}(2, \mathbb{K}[\sqrt{\hbar}])$. Note that $\overline{c d}=c d d_{1} d_{2}=c d$, thus $c d \in \mathbb{K}$.

Let $X=\left(\begin{array}{ll}a & b \\ c & d\end{array}\right) \in G L(2, \mathbb{K}[\sqrt{\hbar}])$ satisfy $\bar{X}=X W D$ with $D=\operatorname{diag}\left(d_{1}, d_{2}\right) \in S O(2, \mathbb{K}[\sqrt{\hbar}])$, where $W=\left(\begin{array}{cc}0 & -1 \\ 1 & 0\end{array}\right)$. The identity is equivalent to the following system: $\bar{a}=b d_{1}, \bar{b}=-a d_{2}, \bar{c}=$ $d d_{1}, \bar{d}=-c d_{2}$. Note that $c d \neq 0$ (otherwise both $c$ and $d$ should be zero). Let $a / c=a^{\prime}+b^{\prime} \sqrt{\hbar}$. Then $b / d=a^{\prime}-b^{\prime} \sqrt{\hbar}$. One can immediately check that $X=R J^{\prime} D^{\prime}$, where $R=\left(\begin{array}{cc}a^{\prime} & b^{\prime} \\ 1 & 0\end{array}\right)$ $\in G L(2, \mathbb{K}), J^{\prime}=\left(\begin{array}{cc}1 & 1 \\ \sqrt{\hbar} & -\sqrt{\hbar}\end{array}\right), D^{\prime}=\operatorname{diag}(c, d) \in \operatorname{diag}(2, \mathbb{K}[\sqrt{\hbar}])$. Note that $\overline{c d}=-c d d_{1} d_{2}=-c d$, thus $c d \in \sqrt{\hbar} \mathbb{K}$.

Lemma 5.7. Let $D \in \operatorname{diag}(M, \overline{\mathbb{K}})$ satisfy $d_{i} d_{M+1-i} \in \mathbb{K}$ for all $i$ (and $d_{n+1}=1$ for $B_{n}$ series; for $C_{n}$ we have $d_{i} d_{M+1-i} \in \sqrt{\hbar} \mathbb{K}$ instead). Then there exists $R \in G L(M, \mathbb{K})$ such that $R J D \in G$.

Proof. Let $X=R J D \in G$. Then $X$ must satisfy $X^{T} S X=S$. Denote $Y:=R^{-1}$. Then $Y S Y^{T}=$ $J D S(J D)^{T}$. Therefore we can find the desired $R$ if and only if the quadratic forms with matrices $S$ and $J D S(J D)^{T}$ are equivalent over $\mathbb{K}$. Note that symplectic forms are always equivalent over a field. Henceforth we consider the cases of $B_{n}$ and $D_{n}$ series. We have

$$
J D S(J D)^{T}=\operatorname{diag}\left(2 d_{1} d_{2 n}, 2 d_{2} d_{2 n-1}, \ldots, 2 d_{n} d_{n+1},-2 \hbar d_{n} d_{n+1}, \ldots,-2 \hbar d_{1} d_{2 n}\right)
$$

for $D_{n}$ and even $n$,

$$
J D S(J D)^{T}=\operatorname{diag}\left(2 d_{1} d_{2 n}, 2 d_{2} d_{2 n-1}, \ldots, d_{n} d_{n+1}, d_{n} d_{n+1}, \ldots,-2 \hbar d_{1} d_{2 n}\right)
$$

for $D_{n}$ and odd $n$, and

$$
J D S(J D)^{T}=\operatorname{diag}\left(2 d_{1} d_{2 n}, 2 d_{2} d_{2 n-1}, \ldots, 2 d_{n} d_{n+1}, 1,-2 \hbar d_{n} d_{n+1}, \ldots,-2 \hbar d_{1} d_{2 n}\right)
$$

for the $B_{n}$ algebra. Thus it suffices to show that for $d \in \mathbb{K}$ the quadratic forms with matrices $\left(\begin{array}{llll}0 & 0 & 0 & 1 \\ 0 & 0 & 1 & 0 \\ 0 & 1 & 0 & 0 \\ 1 & 0 & 0 & 0\end{array}\right),\left(\begin{array}{cccc}d & 0 & 0 & 0 \\ 0 & d & 0 & 0 \\ 0 & 0 & -\hbar d & 0 \\ 0 & 0 & 0 & -\hbar d\end{array}\right)$, and $\left(\begin{array}{cccc}d & 0 & 0 & 0 \\ 0 & d & 0 & 0 \\ 0 & 0 & d & 0 \\ 0 & 0 & 0 & d\end{array}\right)$ are equivalent. This is straightforward.

For $D \in \operatorname{diag}(M, \overline{\mathbb{K}})$ define $T(D)=S D^{-1} S \bar{D}$ (for the $C_{n}$ series we should take $T(D)=-S D^{-1}$ $S^{\prime} \bar{D}$ where $S^{\prime}$ is the matrix with 1's on the antidiagonal, i.e., we "forget" the signs).

We will denote by $\mathbf{Z}$ the set of all diagonal matrices $D=\operatorname{diag}\left(d_{1}, \ldots, d_{M}\right) \in \operatorname{diag}(M, \overline{\mathbb{K}})$ satisfying $d_{i} d_{M+1-i} \in \mathbb{K}$ (also $d_{n+1}=1$ for the $B_{n}$ algebra, $d_{i} d_{M+1-i} \in \sqrt{\hbar} \mathbb{K}$ for the $C_{n}$ algebra) and $D^{-1} \sigma(D) \in C(r)$ for all $\sigma \in \operatorname{Gal}(\overline{\mathbb{K}} / \mathbb{K}[\sqrt{\hbar}])$.

Lemma 5.8. Let $X=R J D \in G, D \in \mathbf{Z}$. Then $X$ is a twisted cocycle if and only if $T(D) \in C(r)$.

Proof. We have $\bar{X}=R J S \bar{D}=X S T(D)$.

Theorem 5.9. We have

$$
\bar{H}_{B D}^{1}(G, r)=\frac{T^{-1}(C(r)) \cap \mathbf{Z}}{C(r) \operatorname{Ker} T}
$$

Proof. For an arbitrary $D \in T^{-1}(C(r)) \cap \mathbf{Z}$ we can find $R \in G L(M, \mathbb{K})$ such that $X=R J D$ is a cocycle. 
Assume that $X=R_{1} J D_{1} \sim Y=R_{2} J D_{2}$. Then $Y=Q X C, Q=R_{2} J D_{2} C^{-1} D_{1}^{-1} J^{-1} R_{1}^{-1}$. Since $Q=$ $\bar{Q}$, we have $D_{2} C^{-1} D_{1}^{-1}=S \overline{D_{2} C^{-1} D_{1}^{-1}} S$ (for $C_{n}$ series one should take $S^{\prime}$ instead of $S$ in the last formula). Thus $D_{2} D_{1}^{-1} \in C(r) \operatorname{Ker} T, D_{2} D_{1}^{-1}=C D_{0}$. If we denote $Q=R_{2} J D_{0} J^{-1} R_{1}^{-1}$, then we have $Y=Q X C$.

Theorem 5.10. Let $r$ be on the list of Lemma 5.3. Then $\left|\bar{H}_{B D}^{1}(G, r)\right|=1$ for $r=r_{D J}$ and $\left|\bar{H}_{B D}^{1}(G, r)\right|=2$ for $r \neq r_{D J}$.

Proof. We will first consider the case of the Drinfeld-Jimbo $r$-matrix. Let $D=\operatorname{diag}\left(d_{1}, \ldots, d_{M}\right)$. Then for $D_{n}$ with even $n$ we have

$$
T(D)=\operatorname{diag}\left(d_{2 n}^{-1} \overline{d_{1}}, d_{2 n-1}^{-1} \overline{d_{2}}, \ldots, d_{1}^{-1} \overline{d_{2 n}}\right),
$$

for $D_{n}$ with odd $n$ we have

$$
T(D)=\operatorname{diag}\left(d_{2 n}^{-1} \overline{d_{1}}, \ldots, d_{n}^{-1} \overline{d_{n}}, d_{n+1}^{-1} \overline{d_{n+1}}, \ldots, d_{1}^{-1} \overline{d_{2 n}}\right),
$$

for $B_{n}$ we have

$$
T(D)=\operatorname{diag}\left(d_{2 n}^{-1} \overline{d_{1}}, d_{2 n-1}^{-1} \overline{d_{2}}, \ldots, d_{n+1}^{-1} \overline{d_{n}}, 1, d_{n}^{-1} \overline{d_{n+1}}, \ldots, d_{1}^{-1} \overline{d_{2 n}}\right),
$$

and for the $C_{n}$ algebra

$$
T(D)=\operatorname{diag}\left(d_{2 n}^{-1} \overline{d_{1}}, \ldots, d_{n+1}^{-1} \overline{d_{n}},-d_{n-1}^{-1} \overline{d_{n+1}}, \ldots,-d_{1}^{-1} \overline{d_{2 n}}\right) .
$$

If $D \in \mathbf{Z}$, then $T(D) \in G$, thus $T(D) \in C(r)$. It is easy to see that an arbitrary matrix in $\mathbf{Z}$ can be decomposed as a product of a matrix in $G$ and a matrix in $\operatorname{Ker} T$. Therefore $\left|H_{B D}^{1}\left(G, r_{D J}\right)\right|=1$. For the $D_{n}$ series decomposing $D \in \mathbf{Z}$ as $D=W D_{1}$ with $W \in \operatorname{Ker} T$ and $D_{1} \in G$ one gets the following: if $D \in \mathbb{K}($ resp. $\mathbb{K}[\sqrt{\hbar}])$, then $W, D_{1} \in \mathbb{K}($ resp. $\mathbb{K}[\sqrt{\hbar}])$.

Now let $r \neq r_{D J}$ (this means that we deal with the case $D_{n}$ for odd $n$ ). Let $D \in \mathbf{Z}$. Then $D=K F$, where $K \in \operatorname{diag}(2 n, \mathbb{K}) \cap \operatorname{Ker} T, F \in G$. Then $F$ is a non-twisted cocycle for $r$ over $\mathbb{K}[\sqrt{\hbar}]$. Hence, because of taking the quotient by $C(r)$ we can assume that either $F \in \operatorname{diag}(2 n, \mathbb{K}[\sqrt{\hbar}])$ or $F=P F_{1}$, where $F_{1} \in \operatorname{diag}(2 n, \mathbb{K}[\sqrt{\hbar}]), P=\operatorname{diag}(1,1, \ldots, \sqrt[4]{\hbar}, 1 / \sqrt[4]{\hbar}, 1, \ldots, 1)$ (see Theorem 4.3). But it is easy to see that $P \in \operatorname{Ker} T$. Therefore in what follows we assume that $D \in G L(2 n, \mathbb{K}[\sqrt{\hbar}])$, $d_{i} d_{2 n+1-i} \in \mathbb{K}$. Finally

$$
\bar{H}_{B D}^{1}(S O(2 n), r)=\frac{T^{-1}(C(r)) \cap \mathbf{Z}}{C(r) \operatorname{Ker} T}=\frac{A}{C(r) \operatorname{Ker} T},
$$

where $A=T^{-1}(C(r)) \cap O(2 n, \mathbb{K}[\sqrt{\hbar}])$.

Recall that we define $\left(d_{1}, \ldots, d_{n}\right)=\operatorname{diag}\left(d_{1}, \ldots, d_{n}, 1 / d_{n}, \ldots, 1 / d_{1}\right)$. We will write the defining relations for $A$ and $C(r)$ in each case. Defining relations for Ker $T$ are $d_{1} \overline{d_{1}}=1, \ldots, d_{n-1} \overline{d_{n}}=$ $1, \overline{d_{n}}=d_{n}$.

Case $1\left(\Gamma_{1}=\left\{\alpha_{n-1}\right\}, \tau\left(\alpha_{n-1}\right)=\alpha_{n}\right)$.

$A:\left(d_{n}^{-1} \overline{d_{n}}\right)^{2}=1$.

$C(r): d_{n}^{2}=1$.

If $d_{n} \in \mathbb{K}$, then $\left(d_{1}, \ldots, d_{n}\right)=\left(d_{1}, \ldots, d_{n-1}, 1\right)\left(1,1, \ldots, d_{n}\right) \in C(r) \operatorname{Ker} T$.

If $d_{n} \in \sqrt{\hbar} \mathbb{K}$, then $\left(d_{1}, \ldots, d_{n}\right)=(1, \ldots, 1, \sqrt{\hbar})\left(1, \ldots, 1, d_{n} / \sqrt{\hbar}\right)\left(d_{1}, \ldots, d_{n-1}, 1\right)$. We have $(1, \ldots, 1) \nsim(1, \ldots, 1, \sqrt{\hbar})$ because if $\left(a_{1}, \ldots, a_{n}\right) \in C(r)$ Ker $T$ then $a_{n} \in \mathbb{K}$. Hence $\bar{H}_{B D}^{1}$ contains two elements.

Case $2\left(\Gamma_{1}=\left\{\alpha_{n}\right\}, \tau\left(\alpha_{n}\right)=\alpha_{n-1}\right)$ is similar to case 1 .

Case 3.1 (Case 3 with $\left.k=n-2: \Gamma_{1}=\left\{\alpha_{n-1}, \alpha_{n-2}\right\}, \tau\left(\alpha_{n-1}\right)=\alpha_{n-2}, \tau\left(\alpha_{n-2}\right)=\alpha_{n}\right)$.

$A:\left(d_{n}^{-1} \overline{d_{n}}\right)^{2}=1 ;\left(d_{n-1} \overline{d_{n-1}}\right)^{2} d_{n}^{-1} \overline{d_{n}}=d_{n-2} \overline{d_{n-2}}$.

$C(r): d_{n}^{2}=1, d_{n-1}^{2} d_{n}=d_{n-2}$.

If $d_{n} \in \mathbb{K}$, then

$$
\left(d_{1}, \ldots, d_{n}\right)=\left(d_{1}, \ldots, d_{n-3}, d_{n-1}^{2}, d_{n-1}, 1\right)\left(1, \ldots, 1, d_{n-2} / d_{n-1}^{2}, 1, d_{n}\right) .
$$

If $d_{n} \in \sqrt{\hbar} \mathbb{K}$, then

$$
\left(d_{1}, \ldots, d_{n}\right)=(1, \ldots, 1, \sqrt{\hbar})\left(d_{1}, \ldots, d_{n-1}^{2}, d_{n-1}, 1\right)\left(1, \ldots, d_{n-2} / d_{n-1}^{2}, 1, d_{n} / \sqrt{\hbar}\right) .
$$


Case 3.2 (Case 3 with $\left.k \neq n-2: \Gamma_{1}=\left\{\alpha_{n-1}, \alpha_{k}\right\}, \tau\left(\alpha_{n-1}\right)=\alpha_{k}, \tau\left(\alpha_{k}\right)=\alpha_{n}\right)$.

To simplify the notation we assume $k=n-3$ (the general case is completely similar) $C(r): d_{n}^{2}=1 ; d_{n-3}=d_{n-2} d_{n-1} d_{n}$.

$A:\left(d_{n}^{-1} \overline{d_{n}}\right)^{2}=1 ; d_{n-3} \overline{d_{n-3}}=d_{n-2} d_{n-1} d_{n}^{-1} \overline{d_{n-2} d_{n-1} d_{n}}$.

If $d_{n} \in \mathbb{K}$, then

$$
\left(d_{1}, \ldots, d_{n}\right)=\left(d_{1}, \ldots, d_{n-2} d_{n-1}, d_{n-2}, d_{n-1}, 1\right)\left(1, \ldots, d_{n-3} / d_{n-2} d_{n-1}, 1,1, d_{n}\right) .
$$

If $d_{n} \in \sqrt{\hbar} \mathbb{K}$, then

$$
\begin{gathered}
\left(d_{1}, \ldots, d_{n}\right)= \\
(1, \ldots, 1, \sqrt{\hbar})\left(d_{1}, \ldots, d_{n-2} d_{n-1}, d_{n-2}, d_{n-1}, 1\right)\left(1, \ldots, d_{n-3} / d_{n-2} d_{n-1}, 1,1, d_{n} / \sqrt{\hbar}\right) .
\end{gathered}
$$

Case $4\left(\Gamma_{1}=\left\{\alpha_{n}, \alpha_{k}\right\}, \tau\left(\alpha_{n}\right)=\alpha_{k}, \tau\left(\alpha_{k}\right)=\alpha_{n-1}\right)$ is similar to case 3 .

\section{CONCLUDING REMARKS}

Consider the isomorphism $\mathfrak{s v}(3,3) \cong \mathfrak{s l}(4)$. Under this isomorphism $F$-points are mapped to $F$-points. Our results imply that for certain $r$-matrices $r_{B D}$ the set $\bar{H}_{B D}^{1}\left(r_{B D}, \mathfrak{s v}(3,3)\right)$ contains 2 elements. On the other hand, $\left|\bar{H}_{B D}^{1}\left(r_{B D}, \mathfrak{s l}(4), G L(4)\right)\right|=1$ (see Ref. 8). This means that we have two twisted Lie bialgebra structures on $\mathfrak{s}(3,3)$ that are non-conjugate but isomorphic. This motivates the following conjecture:

Conjecture 6.1. If $r_{B D}$ is an $r$-matrix from the list of Lemma 5.3 then all twisted Lie bialgebra structures on $g$ corresponding to $r_{B D}$ are isomorphic.

Consider the problem of quantizing Belavin-Drinfeld $r$-matrices. In Ref. 6 Etingof, Schedler, and Schiffmann explicitly quantized the whole Belavin-Drinfeld list. Let us see how this is related to our considerations. Note that any $r$-matrix of twisted type satisfies $r+r^{21}=\sqrt{\hbar} \Omega$, where $\Omega$ is a Casimir element. This means that the quasi-classical limit of the twisted type $r$-matrix is skew. Therefore, Belavin-Drinfeld non-twisted cohomologies parameterize the set of quantizations of a given Belavin-Drinfeld matrix (up to some equivalence that is weaker than isomorphism). Thus, in most cases considered (except for special Belavin-Drinfeld triples for $D_{n}$ ) the Etingof-Schedler-Schiffmann quantization is unique.

\section{ACKNOWLEDGMENTS}

The authors are grateful to J.-H. Lu for valuable suggestions. To the memory of Petr P. Kulish.

${ }^{1}$ Belavin, A. and Drinfeld, V., "Triangle equations and simple Lie algebras," Sov. Sci. Rev., Sect. C: Math. Phys. Rev. 4. 93-165 (1984).

2 Drinfeld, V., "Hopf algebras and the quantum Yang-Baxter equation,” Dokl. Akad. Nauk SSSR 283(5), 1060-1064 (1985).

${ }^{3}$ Etingof, P. and Kazhdan, D., "Quantization of Lie bialgebras I," Sel. Math. 2, 1-41 (1996).

${ }^{4}$ Etingof, P. and Kazhdan, D., "Quantization of Lie bialgebras II," Sel. Math. 4, 213-232 (1998).

${ }^{5}$ Etingof, P. and Schiffmann, O., Lectures on Quantum Groups (International Press, Cambridge, 1988).

${ }^{6}$ Etingof, P., Schedler, T., and Schiffmann, O., "Explicit quantization of dynamical $r$-matrices for finite dimensional semisimple Lie algebras," J. Am. Math. Soc. 13(3), 595-609 (2000).

7 Jimbo, M., "A $q$-difference analogue of $U \mathfrak{g}$ and the Yang-Baxter equation," Lett. Math. Phys. 10, 63-69 (1985).

${ }^{8}$ Kadets, B., Karolinsky, E., Pop, I., and Stolin, A., "Classification of quantum groups and Belavin-Drinfeld cohomologies," Commun. Math. Phys. 344(1), 1-24 (2016).

${ }^{9}$ Kulish, P. P. and Reshetikhin, N. Yu., "Quantum linear problem for the sine-Gordon equation and higher representations," J. Sov. Math. 23, 2435-2441 (1983).

${ }^{10}$ Stolin, A., "Some remarks on Lie bialgebra structures on simple complex Lie algebras," Commun. Algebra 27, 4289-4302 (1999).

${ }^{11}$ Stolin, A. and Pop, I., "Classification of quantum groups and Lie bialgebra structures on $\operatorname{sl}(n, F)$. Relations with Brauer group,” Adv. Math. 293, 324-342 (2016). 